\title{
Type 1 Stickler syndrome: a histological and ultrastructural study of an untreated globe
}

\begin{abstract}
Aims To present a histological and ultrastructural study of an untreated globe in a patient with genetically confirmed type 1 Stickler syndrome.

Methods Histological and electron microscopic examinations were performed on the enucleated globe from the proband of a pedigree with type 1 Stickler syndrome. Linkage analysis was carried out using polymorphic markers flanking the COL2A1 gene and the mutation was identified by direct sequencing.

Results The significant retinal abnormality was incarceration of vitreous collagen within glial strands on the inner surface of an atrophic and gliotic detached retina. The incarcerated collagenous layers contained glial cells and extended from the retina to form strands, some of which contributed to a retrolental membrane. Mutation screening detected a $\mathrm{C}$ to $\mathrm{T}$ mutation in exon 47 that inserted a premature termination codon into the reading frame of the mRNA. Sequence analysis of three of the four affected children confirmed that they were also heterozygous for the base change. The youngest child's DNA was not analysed. Conclusions The study represents the first evidence of abnormal interactions between pathological vitreous collagen and the inner retina in a patient with type 1 Stickler syndrome with a confirmed mutation in the COL2A1 gene.
\end{abstract}

Eye (2006) 20, 1061-1067. doi:10.1038/sj.eye.6702129; published online 2 December 2005

Keywords: Stickler syndrome; pathology; vitreous body; collagen; linkage (genetics); retina
ME MacRae1, DV Patel², AJ Richards³, MP Snead², J Tolmie ${ }^{4}$ and WR Lee ${ }^{5}$

\section{Introduction}

Stickler syndrome (hereditary arthroophthalmopathy, McKusick Nos. 108300 and 184840) is a dominantly inherited disorder of collagen connective tissue ${ }^{1}$ and is the most common inherited cause of rhegmatogenous retinal detachment in childhood with a risk of giant retinal tear which is often bilateral and a frequent cause of blindness. The majority of pedigrees demonstrate a characteristic 'membranous' vitreous anomaly (type 1) associated with mutations in the gene for type II collagen (COL2A1). ${ }^{2-4}$ Other pedigrees exhibit a different (type 2) vitreous phenotype and harbour mutations in the gene encoding the $\alpha 1$ chain of type XI collagen (COL11A1) on chromosome 1.5,6 Other ocular features described include high myopia, drainage angle anomalies, glaucoma, wedge or fleck cataract, and radial retinal paravascular pigmentation. ${ }^{4,7,8}$ Extra-ocular manifestations of the Stickler syndrome are highly variable both between and within kindreds. The most consistently described are the Pierre Robin sequence, mid-facial hypoplasia, sensorineural deafness, joint laxity, and progressive joint degeneration with mild spondyloepiphyseal dysplasia.

Although Stickler syndrome is recognised as being a disease of defective collagen synthesis, the pathological processes taking place in the eye are poorly understood. Studies are restricted to a series published by Blair et a ${ }^{9}$ describing three enucleated eyes from two different pedigrees with Stickler syndrome. Two out of three of these eyes had undergone previous surgical intervention. This work was published prior to the recognition of two different vitreous phenotypes in Stickler syndrome. One ultrastructural study of the
${ }^{1}$ Princess Alexandra Eye Pavilion, Chalmers Street, Edinburgh, UK

${ }^{2}$ Department of Ophthalmology,

Vitreoretinal Service, Addenbrook's NHS Trust, Cambridge, UK

${ }^{3}$ Department of Pathology, University of Cambridge, Cambridge, UK

${ }^{4}$ Duncan Guthrie Institute of Medical Genetics, Yorkhill NHS Trust, Glasgow, UK

${ }^{5}$ University of Glasgow, Gartnavel General Hospital, Great Western Road, Glasgow, UK

Correspondence: ME MacRae, Princess Alexandra Eye Pavilion, Chalmers Street, Edinburgh, EH8 9DR, UK

Tel: +44131536 1674; Fax: + 441315361574 . E-mail: mary.macrae@ doctors.org.uk

Received: 1 April 2005 Accepted in revised form: 26 August 2005 Published online: 2 December 2005 
vitreal membranes in Stickler syndrome has been published $^{10}$ although without associated molecular genetic analysis to confirm subtyping.

We present a histological and ultrastructural study of an untreated globe in a patient with genetically confirmed type 1 Stickler syndrome.

\section{Materials and methods}

\section{Subject}

The proband, aged 19 years, first presented to an ophthalmology department with a total retinal detachment in the left eye. A paediatric ophthalmologist, to whom one of her children had been referred with a squint, subsequently made the diagnosis of Stickler syndrome. The proband is a moderately high myope $(-7.50 /+4.00 \times 90$ OD and $-8.00 /+4.50 \times 100$ OS $)$ with a history of esotropia. Her right eye exhibited the type 1 'membranous' vitreous anomaly and radial paravascular lattice retinopathy. She suffered from arthritis with some restriction of movement in the weight-bearing joints. There was no evidence of midline clefting but audiography confirmed mild sensorineural deafness at all frequencies. To date, the proband has four affected children.

At initial presentation, she was found to have reduced vision in the left eye (measured as 6/60) secondary to a long-standing retinal detachment with proliferative vitreoretinopathy. A flat retinal tear in an area of lattice retinopathy was seen in the right (fellow) eye with vision measured as $6 / 18$. This was treated with external cryotherapy under local anaesthesia and the left eye was managed conservatively in view of the fact that she was 7 months pregnant. A month later she developed a right superotemporal retinal detachment. This was successfully managed surgically.

The left eye remained comfortable until 13 years later, when she returned with a painful blind eye. On examination, there was corneal decompensation with an epithelial defect. Cholesterol crystals coated the iris and formed a pseudohypopyon. There was seclusio pupillae with iris bombé and a mature cataract. The anterior chamber angle was closed causing intractable glaucoma. Pain and failure of medical treatment to control the raised intraocular pressure led to enucleation.

\section{Pathological examination}

The enucleated eye was fixed in buffered glutaraldehyde (2\%) and the PO block was processed through paraffin for routine stains which included hematoxylin and eosin (H\&E), periodic acid Schiff (PAS), Masson trichrome, Perl's stain for iron and the Bodian and Loyez stains for axons and myelin, respectively. Tissue blocks from the retina were processed through Araldite for conventional electron microscopy.

\section{Molecular genetic analysis}

DNA was extracted from venous blood for molecular genetic analysis. Polymorphic markers (D12S331, D12S85, D12S361, D12S347) that flank COL2A1 were used to assess linkage of the disorder in this family to the COL2A1 locus. The mutation was identified by direct sequencing.

\section{Results}

\section{Ocular pathology}

\section{Macroscopic examination}

The dimensions of the globe were $24 \times 26 \times 24 \mathrm{~mm}$ with $5 \mathrm{~mm}$ of optic nerve attached (Figure 1a). The cornea measured $11 \times 12 \mathrm{~mm}$. The anterior chamber contained cholesterol crystals and was shallow; the angle was closed. The lens was opaque and a large white plaque under the anterior capsule impinged on the posterior surface of the iris. The presumed vestigial vitreous persisted as a thin membrane behind the lens; the vitreous gel appeared to be absent. The retina was thickened and detached, with white strands on the inner surface peripherally (Figure 1b). Clumps of pigmentation of various sizes were present on the inner and outer surfaces of the retina.

The choroid was lined internally by large white stellate scars (Figure 1c). The sclera was thinned temporally and the optic nerve appeared to be atrophic.

\section{Microscopic examination}

The corneal epithelium was atrophic, but not significantly oedematous and the endothelium was attenuated. A peripheral pannus was present. The angles were closed, but there was no evidence of goniodysgenesis. An inconspicuous neovascular membrane lined the iris surface and the iris pigment epithelium had migrated onto the anterior surface of the lens capsule. The ciliary body was unremarkable. The lens cortex and nucleus showed non-specific cleft and globule formation behind a thick anterior subcapsular fibrous plaque.

The detached retina was disorganised and gliotic. At the temporal periphery there was a large fibrovascular membrane which incorporated glial cells and was causing a pucker (Figure $2 \mathrm{a}$ and $\mathrm{b}$ ). A membrane, which was continuous with a preretinal membrane, extended towards a retrolental membrane. Whorled clusters of retinal pigment epithelium cells were seen within the 

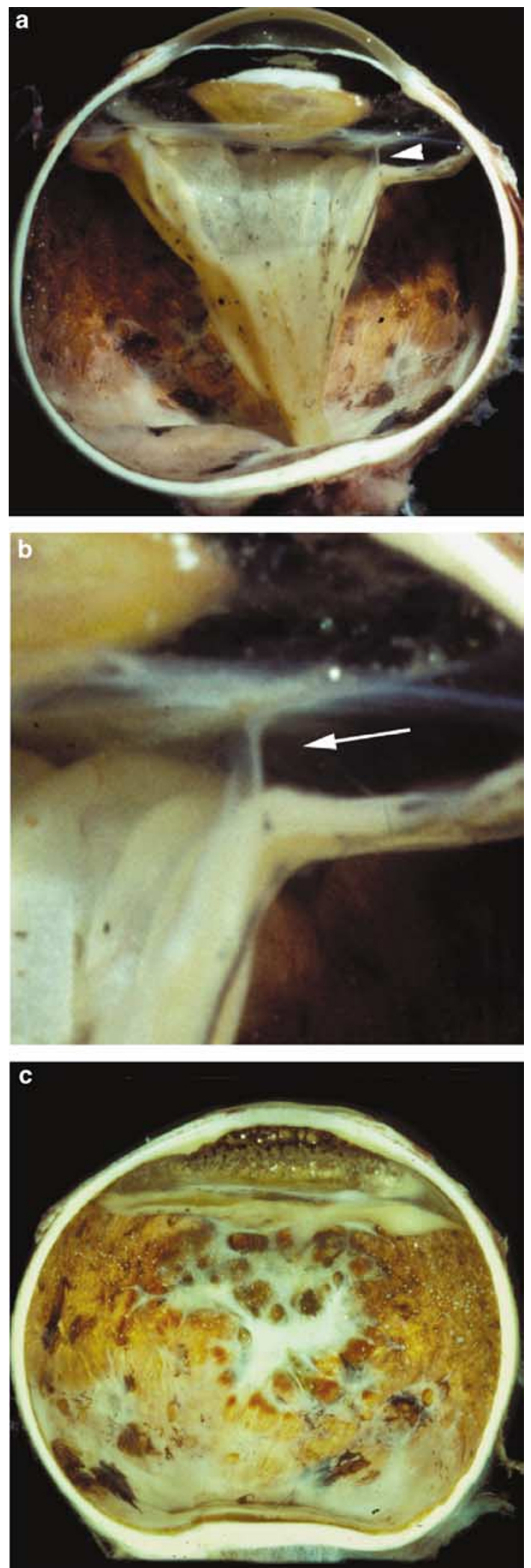

retina (Figure $2 b$ ). At the nasal periphery, a strand of collagen lined by glial cells projected into the vitreous (Figure $2 \mathrm{c}$ and $\mathrm{d}$ ). This strand from the peripheral retina connected with the retrolental membrane, which was formed by loosely arranged fibrous connective tissue containing mononuclear cells (Figure 2e). It was not possible to identify an anterior vitreous face.

Photoreceptors were not identified, and glial cells replaced the outer nuclear layer of the retina. The ganglion cell layer was also replaced by glial tissue but the bipolar cell layer was preserved to some degree (Figure $2 \mathrm{f}$ and $\mathrm{g}$ ). In the middle third of the retina (preequatorial area), the inner retina contained strands of amorphous material that appeared to lie on a corrugated internal limiting membrane. In places this amorphous material, later shown to be collagen on electron microscopy, was divided into two layers by glial cell proliferation (Figure 2f). Projections of glial cells through the internal limiting membrane, some of them with a collagenous core, were a feature identified in the retina closer to the optic nerve head (Figure $2 g$ and $h$ ). The inner and outer nuclear layers were gliotic around an identifiable bipolar layer. There were clumps and clusters of retinal pigment epithelium cells within all layers of the outer retina, in some cases projecting from it into the subretinal space. Adjacent to the disc, the nerve fibre layer retained identifiable axons and myelinated axons were plentiful within the retrolaminar part of the optic nerve (Figure $2 \mathrm{~h}$ ).

The retinal pigment epithelium was atrophic and hypopigmented; calcified drusen were prominent beneath the monolayer. Fibrous strands formed by metaplastic retinal pigment epithelium lined the inner surface of Bruch's membrane; in parts, these contained strips of compact bone. The choroid was atrophic and the stroma contained focal clumps of lymphocytes. The stromal melanocytes were well preserved, but the choriocapillaris was to a great extent replaced by hyalinised connective tissue. A transverse section through the optic nerve revealed axonal loss in the nasal quadrant.

Figure 1 Macroscopic examination of the cross-sectioned globe. (a) The first cut through the globe reveals a closed angle, an anterior subcapsular cataract, an opaque lens and a thin retrolental membrane. Fine strands connect the peripheral retina to the retrolental membrane (arrowhead). Small pigmented flecks are present on both surfaces of the thickened and detached retina. (b) An enlargement of the area shown by the arrowhead in (a) reveals fine strands joining the peripheral retina to the retrolental membrane (arrow). (c) The clumps of pigmentation and large stellate scars on the inner surface of the choroid are demonstrated in the calotte. 

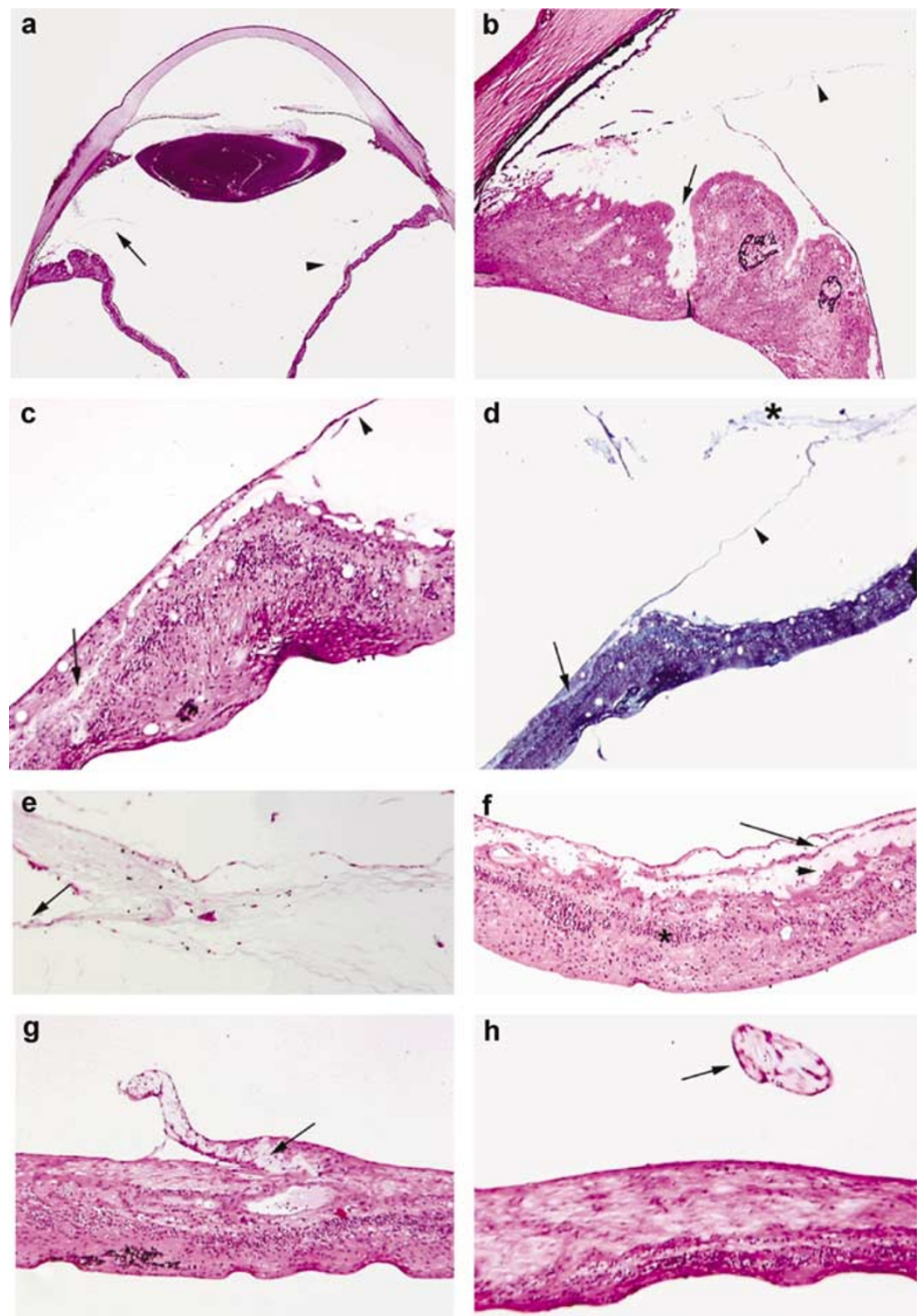

Figure 2 H\&E-stained sections through the anterior segment cut in the horizontal plane (a), the anterior retina (b, c, and e), preequatorial retina $(\mathrm{f})$, posterior retina ( $\mathrm{g}$ and $\mathrm{h}$ ) and Bodian stained retina and retrolental membrane (d). (a) An arrow indicates the temporal retina, an arrowhead the nasal retina $(\times 5)$. (b) The arrow indicates folded retina underlying a fibrovascular membrane temporally. The arrowhead marks a membrane extending from the preretinal membrane to the retrolental membrane $(\times 24)$. (c) On the nasal side, a preretinal glial membrane (arrowhead) extends towards the loose collagenous tissue, which forms the retrolental membrane. The glial membrane is separated from the corrugated inner limiting membrane by an undulating layer of collagen (arrow) $(\times 60)$. (d) Bodian stain demonstrates the connection (arrowhead) between the retinal strand and the retrolental membrane $(*)$. Collagen (arrow) extends into the strand $(\times 24)$. (e) The arrow indicates the limit of the strand shown in $2 \mathrm{c}$ attaching to the retrolental membrane $(\times 150)$. (f) The collagenous tissue layers marked with an arrow and an arrowhead are divided by glial cells $(\times 60)$. $(g)$ The arrow marks projections of glial cells around a collagenous core. A cluster of retinal pigment epithelial cells is seen in the outer retina $(\times 60)$. (h) Preserved axons and photoreceptor nuclei are identifiable and a cross-section of a collagenous strand containing glial cells is present in the subhyaloid space (arrow) $(\times 60)$.

\section{Electron microscopy}

The normal retinal architecture was lost and there was extensive replacement of the tissues of the inner and outer retina by glial cells with migration of retinal pigment epithelial cells (Figure 3a). Occasional macrophages were scattered throughout the gliotic areas. 

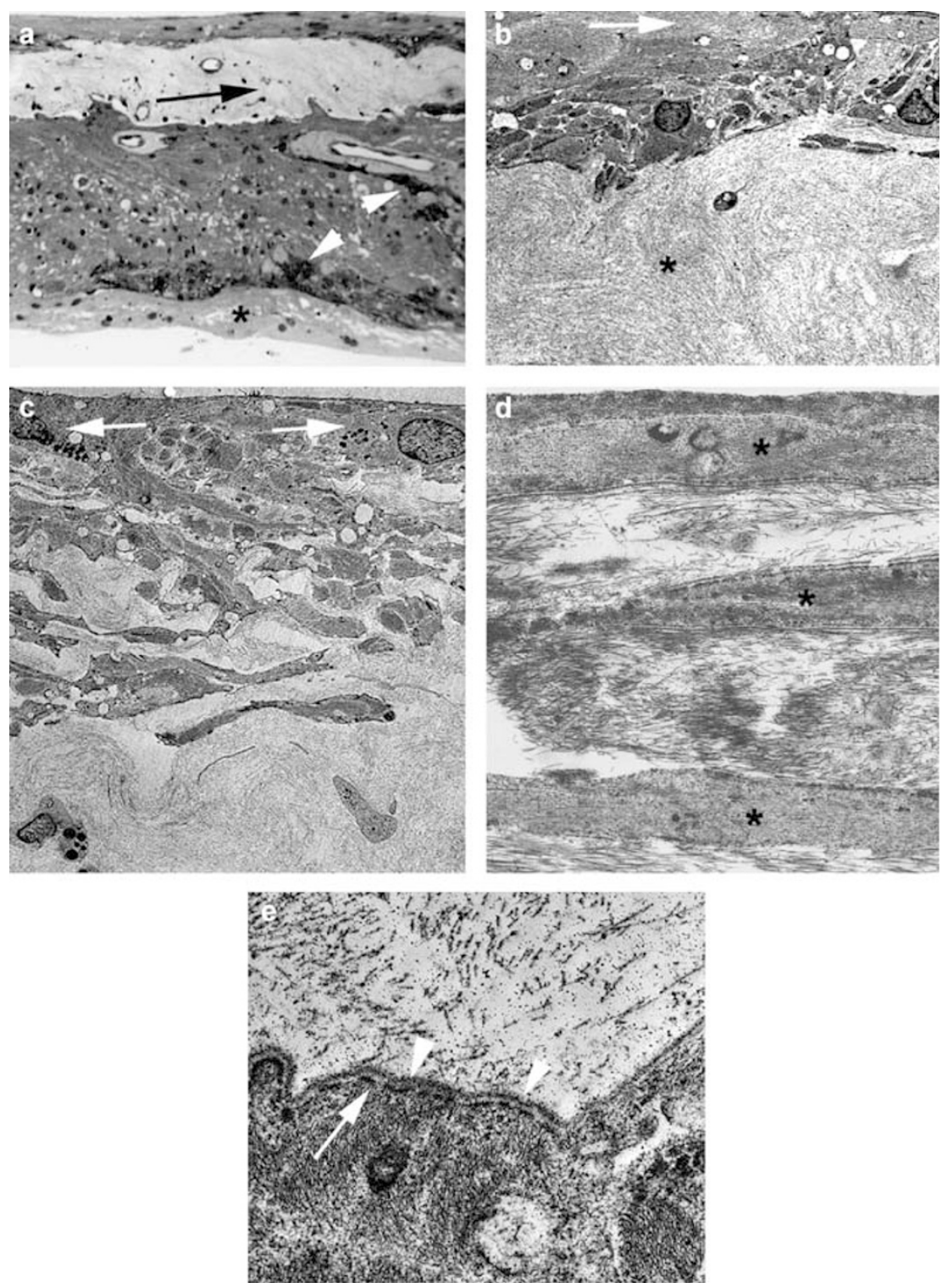

Figure 3 Semithin cross-section (a) and transmission electron micrographs (b-e) of retina. (a) It is possible to identify capillaries within the collagenous tissue (arrow) incarcerated in the gliotic retina. Migration of pigment epithelial cells is a feature (arrowheads). A layer of glial tissue $~_{*}^{*}$ ) lines the outer surface of the retina $(\times 350)$. (b) The glial cells (arrow) form a compact layer on the surface of the whorled collagen fibres $\left(^{*}\right)(\times 3700)$. (c) In some areas, the glial cells are more randomly dispersed and are surrounded by macrophages containing melanin (arrows) $(\times 11000)$. (d) Glial cells contain abundant intermediate filaments $(*)$ which are much finer than the collagen fibres. Their cell membranes are lined by a basement membrane $(\times 25000)$. (e) The cell membrane possesses dense foot processes (arrow) adjacent to a basement membrane (arrowheads). Together, the two layers are identical to the inner limiting membrane of the normal retina $(\times 2300)$.

The majority of the retinal vessels were hyalinised. A thick layer of collagenous material with $65 \mathrm{~nm}$ banding, presumed to be vitreous collagen, was located on the inner limiting membrane of the gliotic retina (Figure $3 b$ ). The glial cells lining the incarcerated collagen were either compact (Figure $3 b$ ) or were infiltrating between the collagen fibres (Figure 3c). The collagen fibres were densely packed in some regions and loosely arranged in others. The glial cells contained compacted intermediate filaments and the cell membranes exhibited the condensations of foot processes in relation to a basement membrane (Figure $3 \mathrm{~d}$ and e). This architecture is equivalent to the inner limiting membrane of the retina.

\section{Molecular genetic analysis}

The affected mother was heterozygous for three of the four COL2A1 linked markers and each of her four 
affected children inherited the same maternal haplotype for these three markers. Mutation screening detected a $\mathrm{C}$ to $\mathrm{T}$ mutation, in exon 47 that inserted a premature termination codon into the reading frame of the mRNA (Figure 4). This is a common type of mutation in Stickler syndrome. Sequence analysis of three of the four affected children confirmed that they were also heterozygous for the base change. Mutation screening was not carried out on the youngest child's DNA.
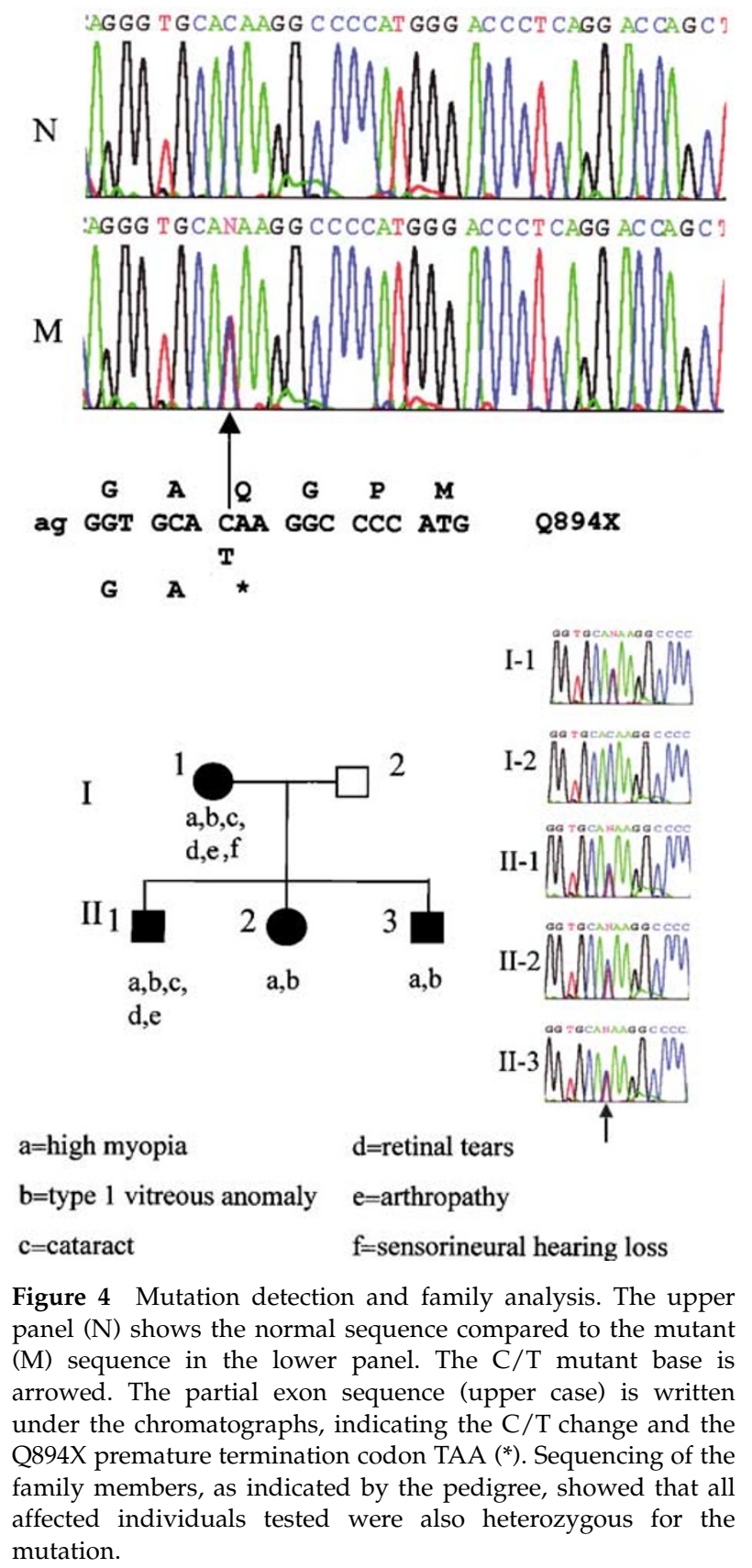

\section{Discussion}

The family described here showed linkage consistent with COL2A1, the mutant locus for most cases of type 1 Stickler syndrome, and mutational analysis confirmed a premature termination codon in exon 47.

The pathology in the present specimen shows many features in common with the series reported by Blair et al. ${ }^{9}$ The globe was larger than average and showed chronic inflammatory changes of the anterior segment with corneal endothelial loss, rubeosis iridis, total angle closure, and lens opacification. An increased global volume has also been recognised clinically during retinal detachment surgery when even non-myopic patients with Stickler syndrome require much larger than average volumes for retinal tamponade.

Many of the other anterior segment features can be considered to be nonspecific, associated with longstanding retinal detachment. Loss of photoreceptors with survival of the nerve fibre layer is a common finding, as is proliferation of retinal pigment epithelium within detached retinas. Glial cell proliferation on the inner limiting membrane of the anterior third of the retina is a well-recognised feature of proliferative vitreoretinopathy, although incarceration of vitreous would be exceptional in rhegmatogenous or traumatic detachment. In this case, the incarcerations extended back to the middle third of the retina but did not involve the posterior third. Only one ultrastructural study of the vitreal membranes in Stickler syndrome has been published ${ }^{10}$ and this revealed cells that possessed cilia, microvilli, cytoplasmic filaments, gap junctions, and basement membrane-like structures; these cells, similar to those observed in the present material, are characteristic of glial cells. The study did not describe extracellular matrix although it is possible that only a small sample was obtained and that there was incarcerated vitreous in the middle third of the detached retina. It is acceptable to regard the incarcerated vitreous and the collagenous strands seen histologically as corresponding to the membranes described clinically. An alternative possibility is that the glial cells formed the collagen as part of the disease process and further studies using immunohistochemistry to identify the collagen type present or advanced morphological techniques to demonstrate messenger RNA for type II collagen in the glial cells would have been helpful. These observations are in contrast to those reported by Blair $e t a l^{9}$ who did not identify incarcerated epiretinal collagen. From the figures provided in their paper, it is not possible to identify the tissues on the inner surface of the retina, although membranes were present. It is possible, however, that the ocular pathology differs between type 1 and type 2 Stickler syndrome. 
In summary, our case describes, in detail, for the first time, the ultrastructural pathology of an untreated globe with long-standing retinal detachment in genetically confirmed type 1 Stickler syndrome. There is a paucity of pathological data available on this disorder. Elucidation of the associated cellular changes and interactions taking place at the vitreoretinal interface, possibly as a result of defective collagen synthesis in the vitreous, provides an important insight into the pathogenesis of retinal detachment in this condition.

\section{Acknowledgements}

The work of the Cambridge group of authors is supported by Action Medical Research

\section{References}

1 Stickler GB, Belau PG, Farrel FJ, Jones JD, Pugh DG, Steinberg AG et al. Hereditary progressive arthroophthalmopathy. Mayo Clin Proc 1965; 40: 433-455.

2 Snead MP, Yates JRW. Clinical and molecular genetics of Stickler syndrome. J Med Genet 1999; 36: 353-359.
3 Snead MP, Payne SJ, Barton DE, Yates JR, Al-Imara L, Pope FM et al. Stickler syndrome: correlation between vitreoretinal phenotypes and linkage to COL 2A1. Eye 1994; 8: 609-614.

4 Richards AJ, Scott JD, Snead MP. Molecular genetics of rhegmatogenous retinal detachment. Eye 2002; 16: 388-392.

5 Martin S, Richards AJ, Yates JR, Scott JD, Pope FM, Snead MP. Stickler syndrome: further mutations in COL11A1 and evidence for additional locus heterogeneity. Eur J Hum Genet 1999; 7: 807-814.

6 Richards AJ, Yates JRW, Williams R, Payne SJ, Pope FM, Scott JD. A family with Stickler syndrome type 2 has a mutation in the COL11A1 gene resulting in the substitution of glycine 97 by valine in alpha 1 (XI) collagen. Hum Mol Genet 1996; 5: 1339-1343.

7 Nielson CE. Stickler's syndrome. Acta Ophthalmol (Copenhagen) 1981; 59: 286-295.

8 Seery CM, Pruett RC, Liberfarb RM, Cohen BZ. Distinctive cataract in the Stickler syndrome. Am J Ophthalmol 1990; 110: 143-148.

9 Blair NP, Albert DM, Liberfarb RM, Hirose T. Hereditary progressive arthro-ophthalmopathy of Stickler. Am J Ophthalmol 1979; 88: 876-888.

10 Miyashita K, Tokunaga M, Akiyama K, Tani M, Ando Y, Chin $\mathrm{S}$ et al. Electron microscopic study on the vitreous membrane of the Stickler syndrome. J JPN Ophthalmol Soc 1994; 98: 86-91. 